Document downloaded from:

http://hdl.handle.net/10251/83422

This paper must be cited as:

Giner Sanz, JJ.; Ortega Navarro, EM.; Pérez Herranz, V. (2016). Optimization of the Perturbation Amplitude for Impedance Measurements in a Commercial PEM Fuel Cell Using Total Harmonic Distortion. Fuel Cells. 16(4):469-479. doi:10.1002/fuce.201500141.

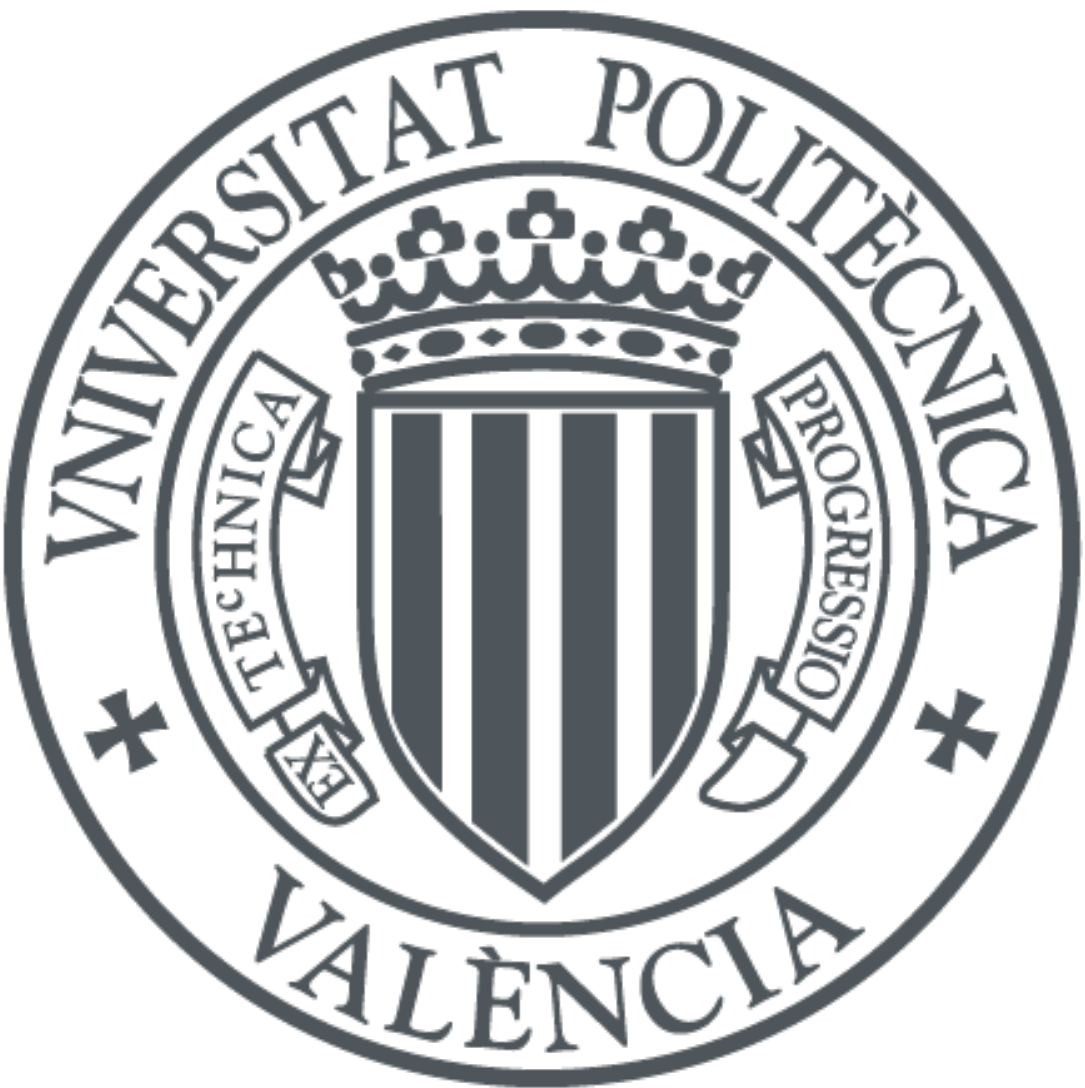

The final publication is available at

http://doi.org/10.1002/fuce.201500141

Copyright Wiley

Additional Information 


\title{
Optimization of the perturbation amplitude for impedance measurements in a commercial PEM fuel cell using total harmonic distortion
}

\author{
J. J. Giner-Sanz, E. M. Ortega, V. Pérez-Herranz* \\ IEC group, Depto. Ingeniería Química y Nuclear, Universitat Politécnica de Valencia \\ Camino de Vera S/N, 46022 Valencia, Spain \\ *Corresponding author. Tel.: +34-96-3877632; fax: +34-96-3877639; \\ E-mail address: vperez@iqn.upv.es (V.Pérez-Herranz)
}

\begin{abstract}
:
One of the most important measurement parameters in electrochemical impedance spectroscopy (EIS) is the perturbation amplitude. The optimum perturbation amplitude value corresponds with a balance between the signal-to-noise ratio improvement and the reduction of the harmonic generation due to nonlinear effects: Therefore, the optimum perturbation amplitude is the maximum amplitude that ensures a linear response of the system. Two strategies were considered in this work: a constant amplitude strategy and a frequency dependent amplitude strategy. On the one hand, for the constant amplitude strategy, the optimum perturbation amplitude for EIS measurements of an individual cell of a commercial PEM fuel cell stack was determined. In order to fulfill this aim, the impedance spectra (at different DC currents) of the individual cell of the commercial PEM fuel cell stack were measured using different perturbation amplitudes. The total harmonic distortion of the recorded voltage signal was determined in each case, applying a FFT based method. The optimum amplitude for each DC current corresponds to the amplitude that minimizes the critical total harmonic distortion value. On the other hand, for the frequency dependent amplitude strategy, the optimum amplitude at each frequency for EIS measurements of an individual cell of a commercial PEM fuel cell stack was determined.
\end{abstract}

Keywords: PEM Fuel Cells, Electrochemical Impedance Spectroscopy, Perturbation Amplitude, Total Harmonic Distortion. 


\section{Introduction}

Electrochemical impedance spectroscopy (EIS) is an electrochemical method widely used nowadays to study electrochemical systems [1-3]. It has been applied in a broad range of application fields [1]. For instance it has been extensively used in the study of fuel cells [4-7], batteries [8-12], supercapacitors [13-17], electrochemical reaction kinetics [18-21], coatings [22-25], corrosion [26-29] and electrochemical sensors [3033]. This electrochemical measurement technique has also been used in fields that are not historically linked to electrochemistry as enzymatic kinetics [34], cancer detection [35], biochemistry [36-37] and immunology [38-39] amongst others. The large spectrum of fields in which EIS has been successfully applied is explained by the fact that this electrochemical method allows to deconvolve, in the frequency domain, the individual contributions of the different physicochemical processes undergoing in the studied system [40].

In current days, EIS is considered as one of the quintessential diagnostic and research tools in the proton exchange membrane fuel cell (PEMFC) field [3]. This is due to the fact that this electrochemical method allows determining the different resistances that arise in a PEMFC, related to different electrochemical and transport processes [1]. This technique provides detailed information on the conductivity of the membrane, on the electrochemical electrode processes and on the transport processes of the system [4143]. All these data are crucial in order to tackle some of the most challenging actual issues of fuel cells, such as membrane drying and gas diffusion layer flooding [44-45]. Therefore, EIS has widely been applied for membrane electrode assembly optimization [46-51]; operation conditions optimization [52-55]; control [56] and diagnosis [57-58]. The technique has been applied both, to fuel cell single cells [58-62] and to fuel cell stacks $[57,63]$.

This electrochemical method consists in the application of a sinusoidal electric perturbation (current or voltage) to the studied system; and the measurement of the generated output electrical signal (voltage or current) [2]. This is repeated for different frequencies of the perturbation signal. For each one of these frequencies, the impedance of the system is determined using generalized Ohm's law [1]:

$$
Z(\omega)=\frac{\mathcal{F}[U(t)]}{\mathcal{F}[I(t)]}
$$

Where $Z(\omega)$ denotes the impedance of the system at angular frequency $\omega ; \mathcal{F}$ stands for the Fourier transform operator; $U(t)$ denotes the potential signal in the time domain; and $I(t)$ is the current signal in the time domain. Therefore, the impedance concept is a generalization of the DC electric resistance concept: it quantifies both, the electric 
resistance of the system (amplitude relation between the current and voltage signals); and the phase difference between both signals.

Four conditions must be met so that the complex Ohm's law, and thus the impedance concept, are valid: causality, finiteness, linearity and stability [64]. If any of these conditions is not fulfilled the obtained impedance spectra may be misleading and unusable to extract proper conclusions on the system [65].

A linear system is a system for which the superposition principle holds: the response to the sum of individual perturbations is the sum of the individual responses to each one of these perturbations. When a sinusoidal perturbation is applied to a linear system, the obtained response signal is a sinusoidal signal of the same frequency than the perturbation [66]. The non-fulfilment of the linearity condition leads to the generation of non-fundamental harmonics, which distort the obtained EIS spectra and bias the results extracted from those spectra [67].

Electrochemical systems, such as PEMFCs, are in general nonlinear systems since they are governed by Butler-Volmer's equation [68]. Consequently, in order to achieve linearity in this kind of systems, the amplitude of the applied perturbation has to be small enough [69]. However, small amplitude perturbation signals lead to low signal-tonoise ratios [1]. Therefore, the quality of an EIS measurement is determined by a tradeoff between the linearity of the system and the signal-to-noise ratio of the signal [70]. On the one hand, very low amplitude perturbations guarantee the linearity of the system but lead to large measurement errors due to a low signal-to-noise ratio. On the other hand, high amplitude perturbations lead to high signal-to-noise ratios, but they generate harmonics in the response signal due to the nonlinearity of the system. These harmonics introduce a systematic error in the impedance spectra measurement, due to the non-fulfilment of the linearity condition.

One of the key parameters of an EIS measurement is the amplitude of the applied perturbation. This amplitude has to be small enough in order to avoid non linear effects; but it should be big enough in order to have a good signal-to-noise ratio [71]. Therefore, the optimum perturbation amplitude can be defined as the maximum perturbation amplitude that ensures a linear response of the system. This optimum amplitude may vary from one system to another [72]; and for a given system, it may vary from one frequency to another [73].

Gode proposed a perturbation amplitude of $5 \%$ of the DC current for galvanostatic EIS measurements in fuel cells [48]; since then, this value has been widely used in literature [57-58]. 
The aim of this work is to determine the optimum perturbation amplitude for EIS measurements of a commercial PEM fuel cell, and verify if it corresponds to the widely used $5 \%$ value. Two strategies were considered in this paper: a constant amplitude strategy and a frequency-dependent amplitude strategy. 


\section{Harmonic analysis and total harmonic distortion}

As stated previously, when a mono-frequency sinusoidal perturbation is applied to a linear system, the response signal is a mono-frequency out of phase sinusoidal signal of the same frequency and different amplitude. According to generalized Ohm's law, the amplitude ratio defines the system's impedance modulus; and the time shift defines the system's impedance argument [1]. However, in the case of nonlinear systems the response signal is a multi-frequency signal: the response signal will correspond with a superposition of different sine waves of different frequencies [70].

Therefore, in electrochemical impedance spectroscopy, if the linearity condition is not satisfied, the response signal will be a superposition of sinusoidal signals of different frequencies (non fundamental components) and a sinusoidal signal of the same frequency that the perturbation signal (fundamental component) [67]. The frequencies of the superimposed signals correspond to integer multiples of the fundamental component frequency: a nonlinear system generates non fundamental harmonics in the response signal [72]. Consequently, the nonlinearity of the system is responsible for the harmonic generation in the response signal: thus, the analysis of the response of the system in the frequency domain is a good quantitative method for evaluating the linearity of the system [2]. The harmonic generation causes a distortion of the measured impedance spectra that may lead to biased or even misguided conclusions [74]. For this reason, it is of utmost importance to maintain the harmonic generation under neglectable levels: this is achieved by using low enough perturbation amplitudes [66].

A great number of theoretical works that demonstrate the generation of nonfundamental harmonics due to the nonlinearity of the system during electrochemical impedance measurements can be found in literature: for instance, the works of Darowicki and coworkers [69], Diard and coworkers [75], Van Gheem [76] and Victoria and Ramanathan [66].

The harmonic generation due to the nonlinearity of the system is the basis of the linearity assessment methods based on harmonic analysis, selected for the present work. These methods are based in the analysis of the response signal in the frequency domain: the analysis of the harmonic content of the response signal of the system can be used to quantify the nonlinearity of the system.

Total harmonic distortion $(\mathcal{T H} \mathcal{H})$ is a parameter that is widely used for harmonic level quantification in electric signals [77]. Its use is widespread in the electrical engineering field; and it is one of the most common quality control parameters for power supply. Since the harmonic content of the response signal of the system can be used to quantify 
the nonlinearity of the system, the total harmonic distortion is a good parameter to quantify the nonlinearity of the system. For this reason, this parameter was selected for this work.

Two different definitions of the $\mathcal{T H \mathcal { D }}$ appear in literature. They differ in the reference they consider. On the one hand, one definition considers the fundamental component as the reference; whereas, the other definition considers the root mean square (rms) value of the signal as the reference. Shmilovitz showed that the first definition is a better measure of the harmonic content of an electrical signal [78]. For this reason, in this work the following definition of the total harmonic distortion of electrical signal $X$ was considered:

$$
\mathcal{J H} \mathcal{D} X=\frac{1}{|\widehat{X}|_{1}} \cdot \sqrt{\sum_{i=2}^{+\infty}|\widehat{X}|_{i}^{2}}
$$

Where $|\hat{X}|$ denotes the amplitude of the Fourier transform of signal $X$ (signal in the frequency domain). Subscript 1 stands for the fundamental component; while subscripts greater or equal to 2 are associated to non-fundamental harmonics.

This parameter was used in this work to quantify the level of harmonics in the response signals during the EIS measurements. In this work the EIS measurements were performed in galvanostatic mode. Therefore, the response signal corresponds with the voltage signal. Thus, the particularization of Eq. (2) to this situation gives the following expression:

$$
\mathcal{T H \mathcal { D } U}=\frac{1}{|\widehat{U}|_{1}} \cdot \sqrt{\sum_{i=2}^{+\infty}|\widehat{U}|_{i}^{2}}
$$

In general, the total harmonic distortion results are expressed as a percentage (of the fundamental component) [78]. 


\section{Methodology}

The aim of this work is to obtain the optimum perturbation amplitude for EIS measurements of an individual cell of a commercial PEMFC stack. The common approach used in literature to tackle this problem is to study the effect of the perturbation amplitude on the impedance spectrum: the biggest perturbation amplitude that does not affect significantly the measured spectrum is selected according to this approach. This strategy was used for example by Yuan and co-workers [58]. However, this method has several weaknesses. Firstly, the electrochemical impedance spectrum may vary because of other causes than the perturbation amplitude (i.e. non stationarity). Consequently, a suboptimal amplitude may be selected because a variation in the impedance spectrum was observed, but that variation was due to other causes than the perturbation amplitude. Secondly, "affect significantly" is quite fuzzy: the criterion to distinguish a significant distortion due to the perturbation amplitude from the inherent variability of the measurement is quite unclear, and it is generally left to the subjectivity of the annalist. Finally, this kind of approach is only useful for a constant amplitude (frequency independent) strategy; but it cannot be used for a frequency dependent amplitude strategy, in which a different amplitude is selected for each frequency. The harmonic analysis method overcomes all these limitations. For this reason, in this work, a $\mathcal{T H \mathcal { D }}$ based method was selected in order to fulfill the fixed goal. Two strategies were considered: a constant amplitude strategy and a frequency dependent amplitude strategy.

In order to achieve this work's objective, the galvanostatic impedance spectra (at different DC currents) of an individual cell of a commercial PEMFC stack were measured using different perturbation amplitudes. In each case, and for each excited frequency, the response signal (the voltage signal) in the time domain was sampled, $U$. A Fast Fourier Transform was applied in order to obtain the response signal in the frequency domain, $\widehat{U}$. Using Eq. (3), the total harmonic distortion of the response signal was calculated for each excited frequency in each measured impedance spectrum. For each measured impedance spectrum, the critical parameter was determined using the following expression:

$$
\mathcal{T H} \mathcal{H} \mathcal{D} U_{c}=\max _{i \in\left\{1 ; 2 ; \cdots ; N_{f}\right\}} \mathcal{T H} \mathcal{H} U_{i}
$$

Where $N_{f}$ stands for the number of frequencies at which the impedance was measured. The frequency associated with the parameter $\mathcal{T H} \mathcal{H} U_{c}$ is the critical frequency of the system. 
The global methodology applied to each obtained spectrum is summarized in figure 1. This methodology can be easily automated in any programming language. In this work, it was fully implemented in Labview $®$. The developed Labview $®$ program takes as input the output of NOVA $囚$, which is the software used to control the impedance measurement system, as it will be explained in the experimental work section. The NOVA®'s output consists in the raw response signal (voltage signal) in the time domain. The implemented Labview $®$ program reads the raw signal in the time domain, calculates the associated signal in the frequency domain using an FFT algorithm; and once, the signal in the frequency domain has been obtained, it calculates the total harmonic distortion using equation (3). There are some commercial softwares available in the market that determine the total harmonic distortion values automatically during the measurement. One of these programs is Thales ${ }^{\circledR}$, from the German company Zahner ${ }^{\circledR}$ [79].

Regardless if the methodology is implemented in a programming language from scratch or if one of the commercial softwares is used, the obtained results are the same: the $\mathcal{T H} \mathcal{D}$ curves and the critical parameters. On the one hand, the critical parameters are used for the constant amplitude strategy, while the $\mathcal{T H} \mathcal{H}$ curves are used for the frequency dependent amplitude strategy.

In the case of the constant amplitude strategy, for a given polarization current, this methodology was applied to each perturbation amplitude: obtaining the critical parameter curve, which corresponds with the curve that represents the $\mathcal{T H} \mathcal{H} U_{c}$ parameter versus the perturbation amplitude for that polarization current. The optimum amplitude for each DC current corresponds with the perturbation amplitude that minimizes the critical $\mathcal{T H} \mathcal{H}$ value.

Figure 2 shows an example of critical parameter curve for a highly nonlinear system: a cathodic electrode of an alkaline electrolyser [71]. Two clear different trends can be observed. For low amplitudes, an increase in the amplitude causes a decrease in the critical parameter of the output signal, $\mathcal{T H} \mathcal{H} U_{c}$, whereas at medium and high amplitudes, an increase in the amplitude leads to an increase of parameter $\mathcal{T H} \mathcal{H} U_{c}$. Therefore, the optimum perturbation amplitude can be identified as the amplitude at which the trend of the critical parameter curve inverts. This curve shape is explained by two antagonistic effects that happen when the perturbation amplitude is increased. On the one side, an increase in the amplitude improves the signal-to-noise ratio, which causes a drop in $\mathcal{T H} \mathcal{H} U_{c}$. On the other side, an increase in the amplitude generates higher levels of non fundamental harmonics due to the nonlinear behaviour of the system. For amplitudes below the optimum perturbation amplitude, the first effect dominates over the second one: the harmonic generation due to nonlinearity is neglectable compared with the improvement of the signal-to-noise ratio. Therefore, the 
net effect of an increase of the amplitude of the perturbation for low amplitudes is a drop in the critical parameter. Consequently, the system can be considered to behave quasi-linearly in this amplitude range. In contrast, for amplitudes above the optimum amplitude, the second effect dominates over the first one: the harmonic generation due to nonlinear effects is no longer neglectable compared to the signal-to-noise ratio improvement. Therefore, the net effect of an increase of the amplitude of the perturbation in this amplitude range is an increase of the critical parameter. Consequently, for perturbation amplitudes above the optimum one, the nonlinear behaviour of the system is significant. These concepts were discussed in detail in a previous work [80].

In the case of the frequency dependent amplitude strategy, the $\mathcal{T} \mathcal{H} \mathcal{D}$ curves were used. These curves correspond with the representation of the $\mathcal{T H} \mathcal{H}$ parameter for each excited frequency for a given spectrum (a given DC current and perturbation amplitude). The optimum amplitude for each frequency corresponds with the perturbation amplitude that minimizes the $\mathcal{T H} \mathcal{H}$ value for that frequency. Therefore, the minimum $\mathcal{T H \mathcal { D }}$ value (with respect to the perturbation amplitude) was identified for each frequency; and the optimum amplitude for that frequency was deduced: it corresponds with the amplitude for which the $\mathcal{T H} \mathcal{H}$ value is minimal for a given frequency. Using this methodology for each frequency at which the impedance was measured, the optimum perturbation amplitude for each frequency was obtained for each DC current. 


\section{Experimental work}

The experimental setup is given by figure 3 . Its main element is a $300 \mathrm{~W}$ commercial PEMFC stack, provided by HeliocentriS $\AA$, composed by 20 individual cells, with an effective area of $58 \mathrm{~cm}^{2}$. The air supply is provided by a compressor and the hydrogen comes from a 200 bar high-pressure storage tank. The humidification of the gas inlets is assured by a humidification system and the fuel cell stack operating temperature is controlled by a refrigeration system. The humidification system consists in two independent bubbling humidification systems, with humidification temperature control. And the refrigeration system consists in a heat exchanger equipped with a continuous pump and a temperature controller. The reactant gases flow rates are controlled using mass flow controllers. The reactant inlet pressures are monitored by pressure gauges and are regulated using manual valves. All the relevant system temperatures are monitored by thermocouples. The overall control was done using a control computer with a Labview $®$ application. All the experiments were carried out in open end anode mode, with constant inlet reactant flow rates: $5 \mathrm{NL} \cdot \mathrm{min}^{-1}$ for the hydrogen stream and $35 \mathrm{NL} \cdot \mathrm{min}^{-1}$ for the air stream. All the experiments were performed in the same operation conditions, given in table 1.

The individual cell galvanostatic impedance spectra were obtained using an Autolab $^{\circledR}$ $302 \mathrm{~N}$ potentiostat/galvanostat with FRA module and $20 \mathrm{~A}$ booster, controlled using NOVA® software. The selected frequency range extended from $5 \mathrm{kHz}$ to $10 \mathrm{mHz}$, with 50 frequencies logarithmically spaced. The spectra were measured for 3 different polarization currents and 10 different perturbation amplitudes for each DC current, as stated in table 1. A maximum perturbation amplitude of $80 \%$ of the DC current was considered in this work in order to prevent that any cell of the PEMFC stack worked as an electrolyser (polarization inversion) in any moment. Table 2 gives the measurement parameters used to perform the EIS measurements.

A 15 min preconditioning was performed before each measurement in order to assure that the initial point was the same in all the experiments. The preconditioning was done at the DC current associated to the experiment that was going to be performed. 


\section{Experimental results analysis and discussion}

\subsection{THD curves}

Figure 4 shows the obtained $\mathcal{T H} \mathcal{H}$ curves $(\mathcal{T H} \mathcal{H}$ U parameter vs the excited frequency) for a DC current of $1 \mathrm{~A}$, and relative perturbation amplitudes of $10 \%$ and $20 \%$. The critical frequency and the critical parameter $\left(\mathcal{T H} \mathcal{H} U_{c}\right)$ for each perturbation amplitude are identified on the graph. The $\mathcal{T H \mathcal { D }}$ curves obtained for the other perturbation amplitudes and the other DC currents are analogous to the ones shown in figure 4.

It can be observed that the critical frequency range of the system (the frequency range for which the harmonic content is higher) is the intermediate frequency range, from 1 $\mathrm{Hz}$ to $100 \mathrm{~Hz}$. This type of $\mathcal{T H \mathcal { D }}$ curve is generally associated with an electric coupling between the system and the electric grid; thus, in this case, the harmonic content of the signal is mainly noise. This indicates that the harmonic generation due to the nonlinearity of the system is neglectable compared to the noise, at least in the intermediate frequency zone. Consequently, the perturbation amplitude is small enough to be able to accept the hypothesis of linearity of the system in this case.

Figure 4 compares the $\mathcal{T H} \mathcal{H}$ curve obtained for a $\mathrm{DC}$ current of $1 \mathrm{~A}$ and a relative perturbation amplitude of $10 \%$ with the $\mathcal{T H \mathcal { D }}$ curve obtained for a DC current of $1 \mathrm{~A}$ and a relative perturbation amplitude of $20 \%$. It can be observed that both curves have the same shape, and present the same peaks at the same frequencies. However, the peak height is lower in the higher amplitude case: an increase in the perturbation amplitude leads to a drop of the peak heights; thus, to a drop in the harmonic content in the response signal. This is due to the improvement of the signal-to-noise ratio when the perturbation amplitude is raised. An increase of the perturbation amplitude leads to an increase in the signal, while the noise remains constant: thus, the signal-to-noise ratio increases and the harmonic content of the signal drops. This is the typical trend in the linear behaviour zone.

Several works that study the generation of nonlinear effects in a PEM fuel cell system are available in literature [81-85]. In particular, Mao and co-workers obtained the theoretical $\mathcal{T H \mathcal { D }}$ curves for a PEM fuel cell [83]. These theoretical $\mathcal{T H} \mathcal{H}$ curves arise due to the generation of nonlinear effects, and therefore are characteristic of the nonlinear zone behaviour. The fact that the $\mathcal{T H \mathcal { D }}$ curves obtained in this work (figure 4) have a completely different overall shape than the theoretical $\mathcal{T H \mathcal { D }}$ curves reaffirms the hypothesis that the system is in its linear behaviour zone, in which the nonlinear effect generation can be considered negligible in comparison to the noise. 


\subsection{Constant amplitude strategy}

\subsubsection{Critical parameter curves}

Figure 5 represents the critical parameter curve for each DC current. The same behaviour is observed for the three DC currents: an increase in the perturbation amplitude causes a drop of the critical parameter. This is due to the improvement of the signal-to-noise ratio with the increase in the perturbation amplitude. Thus, the harmonic generation due to the nonlinearity of the system is neglectable compared to the noise for all the range of perturbation amplitudes explored in this work. Consequently, an increase in the perturbation amplitude improves the signal-to-noise ratio and does not produce significant harmonics due to the nonlinear effects: resulting in a net reduction of the harmonic content of the signal; thus, a drop of the critical parameter.

As stated before, the optimum amplitude for each DC current corresponds to the amplitude that minimizes the critical THD value. In this case, only the first trend of figure 2 can be observed. Therefore, the optimum perturbation amplitude is higher than the maximum amplitude considered in this work. It can be deduced that the optimum relative amplitude for the constant amplitude strategy for the three DC currents explored in this work is the same: $80 \%$ of the DC current. This value is clearly higher than the $5 \%$ value widely used in literature. Such large amplitudes do not cause non linear effects on account of the high linearity of the behaviour of the commercial PEMFC studied in this work. In other words, the studied system is linear enough so that the nonlinear harmonics are neglectable in comparison to the noise even for very high perturbation amplitudes. It should be noted that it is a balance between the nonlinear effects and the noise. Therefore, if the noise in the system was reduced enough so that its magnitude is significantly lower to the nonlinear effects of the system (i.e. avoiding electrical coupling with the grid), the optimum amplitude may change.

\subsubsection{Nyquist plots}

Figure 6 shows the Nyquist plots obtained for 3 different perturbation amplitudes (5\%, $50 \%$ and $80 \%$ ) at a DC current of $1 \mathrm{~A}$. Since the 3 spectra correspond to the same operation conditions, they correspond to 3 measurements of the same "real" spectrum. The effect of the perturbation amplitude can be clearly observed. The 3 spectra are almost identical in the high frequency zone and in the low frequency range; whereas, in the critical frequency zone identified in section $5.1(1 \mathrm{~Hz}-100 \mathrm{~Hz})$, the effect of the perturbation amplitude is very clear. The $5 \%$ spectrum presents a noisy pattern in the critical frequency zone. This is due to the very low signal-to-noise ratio (high $\mathcal{T H} \mathcal{D}$ value) in this frequency range for a perturbation amplitude of $5 \%$, as it was observed in section 5.1. The spectra obtained with perturbation amplitudes $50 \%$ and $80 \%$ are almost 
identical except in the frequency range $68.87 \mathrm{~Hz}-23.60 \mathrm{~Hz}$, where they differ significantly. These observations are consistent with the trends identified in the $\mathcal{T H} \mathcal{D}$ curves in section 5.1 .

The critical frequency zone corresponds with the frequency zone where the spectrum is more sensible to perturbation amplitude changes, since this zone corresponds with the zone with lowest signal-to-noise ratios (highest $\mathcal{T H \mathcal { D }}$ values): the perturbation amplitude has to be high enough in order to overcome the intense noise in this critical frequency zone. For low and high frequencies, since the noise is low, the obtained spectra even for low perturbation amplitudes do not show the noisy pattern.

\subsection{Frequency dependent amplitude strategy}

Figures 7 and 8 show the detail for low and high frequencies of the THD curves (THDU parameter vs the excited frequency) for relative perturbation amplitudes of $10 \%$ and $20 \%$, represented in figure 4 .

In section 5.1, it was observed that in the intermediate frequency range the THD curve peak height is lower in the higher amplitude case: an increase in the perturbation amplitude leads to a drop of the harmonic content in the response signal. It was deduced from this observation that the system behaves quasi-linearly in the intermediate frequency zone for perturbation amplitudes of $10 \%$ and $20 \%$.

In figures 7 and 8 , it can be observed that for low frequencies and high frequencies the trend is reversed: the THD curve is higher in the higher amplitude case. An increase in the perturbation amplitude leads to an increase of the THD parameter for high and low frequencies; thus, to an increase in the harmonic content in the response signal. This is due to the generation of non-fundamental harmonics because of the non-linearity of the system: an increase in the perturbation amplitude leads to a higher generation of non fundamental harmonics, and thus to a response signal with a higher harmonic content. This is the typical trend in the non-linear behaviour zone [71]. It can be deduced that the system behaves nonlinearly in the high and low frequency zones for perturbation amplitudes of $10 \%$ and $20 \%$ : in these frequency zones, the non-linear effects dominate on the signal-to-noise ratio improvement.

Therefore, this observation shows that a given system can behave linearly in a frequency zone and non-linearly in another frequency zone for a given perturbation amplitude. This observation motivates the need to consider a frequency-dependent perturbation amplitude: large perturbation amplitudes may be applied in the linear frequency zone (in order to improve as much as possible the signal-to-noise ratio); and low perturbation amplitudes have to be applied in the non-linear frequency zone (in order to avoid 
distortions of the spectrum due to non-linear effects). In other words, the sensibility of a system to non-linear effects is frequency dependent.

Figure 9 presents the optimum relative amplitude for each frequency. It can be observed that the optimum amplitude in the intermediate frequency zone is significantly higher (up to $80 \%$ of the DC current) that the optimum amplitude in the high and low frequency zones. This is consistent with the conclusions drawn from figures 4, 7 and 8 . On the one hand, in the intermediate frequency zone, the system behaves as a quasi-linear system, and the noise in the system is very high (due to couplings between the system and the electric grid): a large perturbation amplitude should be used in this frequency range in order to improve as much as possible the signal-to-noise ratio without generating significant non-linear effects. On the other hand, because the system behaves moderately as a non-linear system in the low and high frequency zones, lower perturbation amplitudes have to be used in these frequency ranges in order to avoid the generation of non-fundamental harmonics. However, even in these frequency zones the optimum amplitude is of $10 \%$, which is twice the perturbation amplitude recommended in bibliography.

Finally, figure 10 compares the Nyquist plots obtained for a DC current of $1 \mathrm{~A}$ using the frequency independent strategy with an amplitude of $80 \%$ (Refer to section 5.2) and using the frequency dependent strategy (Refer to figure 9). It can be observed, that with the exception of the $52.70 \mathrm{~Hz}$ point, both spectra are almost identical. The $52.70 \mathrm{~Hz}$ point corresponds with an anomalous point due to resonance with the electric grid (50 $\mathrm{Hz}$ ); therefore, it can be deduced that both strategies lead to the same spectrum. So, even if the system exhibits nonlinear behaviour in the high and low frequency zones, the nonlinear behaviour is not important enough in order to affect significantly the obtained spectrum. Consequently, in this case, the frequency dependent strategy does not improve any further the measurement obtained using the frequency independent strategy with optimum perturbation amplitude. So even if from a theoretical point of view the frequency dependent strategy should be used (since the systems exhibits linear/nonlinear behaviour in different frequency zones); from a practical point of view, the frequency independent strategy gives the optimum spectrum. In other systems (or in this system and other operation conditions), in which the nonlinear effects are stronger than in this case, the use of the frequency dependent strategy may be required in order to obtain the optimum spectra. 


\section{$\underline{\text { 6. Conclusions }}$}

In conclusion, the presented $\mathcal{T H \mathcal { D }}$ based method was successfully used to obtain the optimum perturbation amplitude for EIS measurements of an individual cell of a commercial PEMFC stack for two strategies: a frequency independent perturbation amplitude strategy and a frequency dependent perturbation amplitude strategy.

For the frequency independent perturbation amplitude strategy the optimum relative amplitude is $80 \%$ of the DC current.

For the frequency dependent perturbation amplitude strategy: Since the system behaved quasi-linearly in the intermediate frequency zone, the optimum perturbation amplitudes in this frequency zone are high (up to $80 \%$ of the DC current). In contrast, the optimum perturbation amplitudes are significantly lower in the low and high frequency zones, where the system behaved non-linearly. However, even in the low and high frequency zones, the optimum amplitude is $10 \%$ of the DC current.

Consequently, it can be deduced that the literature $5 \%$ value is a very conservative value. Using this value may lead to obtaining a spectrum of non-optimum quality. Therefore, authors recommend the optimization of the perturbation amplitude for each system, rather than using the $5 \%$ value given in literature. Moreover, since it has been shown that a given system may present quasi-linear and non-linear behaviour in different frequency zones for a given perturbation amplitude, a frequency dependent perturbation amplitude is highly recommended. Even if in some cases (i.e. the case presented in this work), the frequency-dependent perturbation amplitude strategy will not improve further the obtained spectra with respect to the frequency independent perturbation amplitude strategy with optimum amplitude. 


\section{Nomenclature}

\section{Normal letters}

$f_{c} \quad$ Critical frequency $(\mathrm{Hz})$

$I_{D C} \quad$ Polarization current (A)

$N_{f} \quad$ Number of frequencies

$\mathcal{T H \mathcal { H }} \quad$ Total harmonic distortion (\%)

$\mathcal{T H} \mathcal{H} U \quad$ Total harmonic distortion of the voltage signal (\%)

$\mathcal{T H} \mathcal{H} U_{c} \quad$ Total harmonic distortion for the critical frequency (\%)

$X \quad$ Signal in time domain

$\hat{X} \quad$ Signal in frequency domain (Fourier transform)

\section{Greek letters}

$\Delta I \quad$ Galvanostatic perturbation amplitude $(A)$ 


\section{Acknowledgments}

The authors are very grateful to the Generalitat Valenciana for its economic support in form of Vali+d grant (Ref: ACIF-2013-268). 


\section{References}

[1] M.E. Orazem, B. Tribollet, Electrochemical impedance spectroscopy, John Wiley \& Sons, New Jersey, 2008.

[2] E. Barsoukov, J.R. Macdonald, Impedance spectroscopy. Theory, experiment and applications, John Wiley \& Sons, New Jersey, 2005.

[3] A. Iranzo, M. Muñoz, F.J. Pino, F. Rosa, Journal of Power Sources 2011, 196, 4264.

[4] F. Wang, F.X. Miao, W.B. Guan, Fuel Cells 2015, 15, 427.

[5] A. Baricci, M. Zago, A. Casalegno, Fuel Cells 2014, 14, 926.

[6] Y.S. Chung, H. Kim, H.C. Yoon, J.S. Chung, N.M. Sammes, Fuel Cells 2015, 15, 416.

[7] B. Wahdame, D. Candusso, X. François, F. Harel, A. De Bernardinis, J.M. Kauffmann, G. Coquery, Fuel Cells 2007, 7, 47.

[8] N. Penazzi, S. Bodoardo, R. Bongiovanni, C. Gerbaldi, G. Meligrana, G. Mulas, J. Nair, Fuel Cells 2009, 9, 273.

[9] S.M.M Alavi, C.R. Birkl, D.A. Howey, Journal of Power Sources 2015, 288, 345.

[10] J. Huang, Z. Li, J. Zhang, Journal of Power Sources 2015, 273, 1098.

[11] Y.J. Cho, I.J. Park, H.J. Lee, J.G. Kim, Journal of Power Sources 2015, 277, 370.

[12] T. Feng, L. Yang, X. Zhao, H. Zhang, J. Qiang, Journal of Power Sources 2015, 281, 192.

[13] M.S. Javed et al., Journal of Power Sources 2015, 285, 63.

[14] S. Ghasemi, F. Ahmadi, Journal of Power Sources 2015, 289, 129.

[15] S. Sathyamoorthi, V. Suryanarayanan, D. Velayutham, Journal of Power Sources 2015, 274, 1135.

[16] V. Sedlakova et al., Journal of Power Sources 2015, 286, 58.

[17] S.H. Lee, S.G. Lee, J.R. Yoon, H.K. Kim, Journal of Power Sources 2015, 273, 839. 
[18] N. Wagner, K.A. Friedrich, Fuel Cells 2009, 9, 237.

[19] W. Li et al., Journal of Power Sources 2015, 284, 272.

[20] H. Ghanbarlou, S. Rowshanzamir, E. Kazemisab, M.J. Parnian, Journal of Power Sources 2015, 273, 981.

[21] Z.B. Wang, P.J. Zuo, G.P. Yin, Fuel Cells 2009, 9, 106.

[22] F. Nobili, S. Dsoke, M. Mancini, R. Marassi, Fuel Cells 2009, 9, 264.

[23] F.J. Chen, F. Tao, C.M. Wang, W.L. Zhang, L. Chen, Journal of Power Sources 2015, $285,367$.

[24] P. Swain, M. Viji, P. Mocherla, C. Sudakar, Journal of Power Sources 2015, 293, 613.

[25] J. Cao, G. Hu, Z. Peng, K. Du, Y. Cao, Journal of Power Sources 2015, 281, 49.

[26] M.A. Deyab, Journal of Power Sources 2015, 292, 66.

[27] M.A. Deyab, Journal of Power Sources 2015, 280, 190.

[28] C.S. Ni, L.Y. Lu, C.L. Zeng, Y. Niu, Journal of Power Sources 2014, 261, 162.

[29] T.J. Pan, B. Zhang, J. Li, Y.X. He, F. Lin, Journal of Power Sources 2014, 269, 81.

[30] B. Pejcic, R. De Marco, Electrochimica Acta 2006, 51, 6217.

[31] L. Manjakkal et al., Electrochimica Acta 2015, 168, 246.

[32] R. Pauliukaite, M.E. Ghica, O. Fatibello-Filho, C.M.A. Brett, Electrochimica Acta 2010, 55, 6239.

[33] F. Long, Z. Zhang, J. Wang, Liang Yan, B. Zhou, Electrochimica Acta 2015, 168, 337.

[34] T. Vidakovic-Koch, V.K. Mittal, T.Q.N. Do, M. Varnicic, K. Sundmacher, Electrochimica Acta 2013, 110, 94.

[35] R. Wang, J. Di, J. Ma, Z. Ma, Electrochimica Acta 2012, 61, 179. 
[36] M. Naumowicz, A.D. Petelska, Z.A. Figaszewski, Electrochimica Acta 2009, 54, 1089.

[37] K. Mallaiya, S. Rameshkumar, S.S. Subramanian, S. Ramalingam, T. Ramachandran, Electrochimica Acta 2014, 138, 360.

[38] W.M. Hassen, V. Duplan, E. Frost, J.J. Dubowski, Electrochimica Acta 2011, 56, 8325.

[39] A. Sargent, O.A. Sadik, Electrochimica Acta 1999, 44, 4667.

[40] F. Ciucci, C. Chen, Electrochimica Acta 2015, 167, 439.

[41] T. Kurz, J. Keller, Fuel Cells 2011, 11, 518.

[42] G. Li, P.G. Pickup, Journal of the Electrochemical Society 2003, 150, C745.

[43] X. Yan et al., International Journal of Hydrogen Energy 2007, 32, 4358.

[44] H. Li et al., Journal of Power Sources 2008, 178, 103.

[45] T.V. Nguyen, R.E. White, Journal of the Electrochemical Society 1993, 140, 2178.

[46] K. Su, X. Yao, S. Sui, Z. Wei, J. Zhang, S. Du, Fuel Cells 2015, 15, 449.

[47] S. Latorrata, P. Gallo Stampino, C. Cristiani, G. Dotelli, Fuel Cells 2015, 15, 463.

[48] P. Gode, F. Jaouen, G. Lindbergh, A. Lundblad, G. Sundholm, Electrchimica Acta 2003 , 48, 4175.

[49] M.S. Çögenli, S. Mukerjee, A.B. Yurtcan, Fuel Cells 2015, 15, 288.

[50] T.V. Reshetenko, H.T. Kim, U. Krewer, H.J. Kweon, Fuel Cells 2007, 7, 238.

[51] A.G. Hombradosa et al., Journal of Power Sources 2005, 151, 25.

[52] X. Sun, A.D. Bonaccorso, C. Graves, S.B. Ebbesen, S.H. Jensen, A. Hagen, P. Holappels, P.V. Hendriksen, M.B. Mogensen, Fuel Cells 2015, DOI: 10.1002/fuce.201500020.

[53] R.E. Silva, F. Harel, S. Jemeï, R. Gouriveau, D. Hissel, L. Boulon, K. Agbossou, Fuel Cells 2014, 14, 894. 
[54] H.C. Young, G.S. Yong, C.C., Seong, I.W. Won, S.H. Hak, Journal of Power Sources 2003, 118, 334.

[55] T.J.P Freire, E.R. Gonzalez, Journal of Electroanalitic Chemistry 2001, 503, 57.

[56] S. Keller, A. Popenheim, M. Hempel, K. Gläsker, Y. Manoli, Fuel Cells 2014, 14, 758.

[57] X. Yuan et al., Journal of Power Sources 2006, 161, 920.

[58] F. Chen, Y. Gao, Fuel Cells 2015, 15, 337.

[59] S.J.C Cleghorn et al., Journal of Power Sources 2006, 158, 446.

[60] M. Oszcipok, D. Riemann, U. Kronenwett, M. Kreideweis, M. Zedda, Journal of Power Sources 2005, 145, 407.

[61] A. Mommaa, Y. Kagaa, Solid State Ionics 2004, 174, 87.

[62] J.C. Amphlett, B.A. Peppley, E. Halliop, A. Sadiq, Journal of Power Sources 2001, 96, 204.

[63] T.E. Springer, T.A Zawodzinski, M.S. Wilson, S. Gottesfeld, Journal of the Electrochemical Society 1996, 143, 587.

[64] D.D. Macdonald, Elzbieta Sikora, Electrochimica Acta 1997, 43, 87.

[65] M. Urquidi-Macdonald, S. Real, D.D. Macdonald, Electrochimica Acta 1990, 35, 1559.

[66] S. Noyel Victoria, S. Ramanathan, Electrochimica Acta 2011, 56, 2606.

[67] M. Kiel, O. Bohlen, D.U. Sauer, Electrochimica Acta 2008, 53, 7367.

[68] K. Darowicki, Electroquimica Acta 1997, 42, 1781.

[69] K. Darowicki, Electroquimica Acta 1995, 40, 439.

[70] G.S. Popkirowv, R.N. Schindler, Electroquimica Acta 1995, 40, 2511.

[71] J.J. Giner-Sanz, E.M. Ortega, V. Pérez-Herranz, 6th International Conference on Fundamentals and Development of Fuel Cells (FDFC2015), Toulouse, France, 2015. 
[72] B. Hirschorn, B. Tribollet, M.E. Orazem, Israel Journal of Chemistry 2008, 48, 133.

[73] J.J. Giner-Sanz, E.M. Ortega, V. Pérez-Herranz, 5th European PEFC and H2 Forum (EFCF2015), Lucerne, Switzerland, 2015.

[74] K. Darowicki, Electrochimica Acta 1995, 40, 767.

[75] J.P. Diard, B. Le Gorrec, C. Montella, Journal of Electroanalytical Chemistry 1997, $432,27$.

[76] E. Van Gheem et al Electrochimica Acta 2004, 49, 4753.

[77] J. Arrillaga, N.R. Watson, S. Chaen, Power systems quality assessment, Wiley, New York, 2000.

[78] D. Shmilovitz, IEEE transactions on power delivery 2005, 20, 526.

[79] Zahner, user manual for Thales.

[80] J.J. Giner-Sanz, E.M. Ortega, V. Pérez-Herranz, Electrochimica Acta 2015, 186, 598.

[81] Q. Mao, U. Krewer, R. Hanke-Rauschenbach, Electrochemistry Communications 2010, 12, 1517.

[82] Q. Mao, U. Krewer, R. Hanke-Rauschenbach, Electrochimica Acta 2012, 68, 60.

[83] Q. Mao, U. Krewer, R. Hanke-Rauschenbach, Electrochimica Acta 2013, 103, 188.

[84] T. Kadyk, R. Hanke-Rauschenbach, K. Sundmacher, Journal of Electroanalytical Chemistry 2009, 630, 19.

[85] B. Bensmann, M. Petkovska, T. Vidakovic-Koch, R. Hanke-Rauschenbach, K. Sundmacher, Journal of the Electrochemical Society 2010, 157, B1279. 



\section{$\underline{\text { LIST OF TABLES }}$}

Table 1. Experimental parameters

Table 2. EIS measurement parameters 


\section{$\underline{\text { LIST OF FIGURES }}$}

Figure 1. Methodology applied to each spectrum

Figure 2. Example of a critical parameter curve for a nonlinear system

Figure 3. Experimental setup

Figure 4. Comparison of THD curves obtained with a perturbation amplitude of $100 \mathrm{~mA}$ (10\%) and $200 \mathrm{~mA}(20 \%)$

Figure 5. Critical parameter vs the relative amplitude

Figure 6. Nyquist plots obtained for different perturbation amplitudes at a DC current of $1 \mathrm{~A}$

Figure 7. Detail of the low frequency zone of figure 4

Figure 8. Detail of the high frequency zone of figure 4

Figure 9. Optimum perturbation amplitude for each frequency

Figure 10. Nyquist plots obtained with both strategies at a DC current of $1 \mathrm{~A}$ 
Table 1. Experimental parameters

\begin{tabular}{|c|c|}
\hline Parameter & Value or range of values \\
\hline Operation temperature & $70^{\circ} \mathrm{C}$ \\
\hline $\begin{array}{c}\text { Hydrogen humidification } \\
\text { temperature }\end{array}$ & $70^{\circ} \mathrm{C}$ \\
\hline Air humidification temperature & $70^{\circ} \mathrm{C}$ \\
\hline DC current & $\{0.5 A ; 1 A ; 4 A\}$ \\
\hline Relative perturbation amplitude & $\{2.5 \% ; 5 \% ; 10 \% ; 20 \% ; 30 \% ; \cdots ; 80 \%\}$ \\
\hline
\end{tabular}


Table 2. EIS measurement parameters

\begin{tabular}{|c|c|}
\hline Measurement parameter & Value \\
\hline Integration time & $1.0 \mathrm{~s}$ \\
\hline Number of integration cycles & $1 \mathrm{cycle}$ \\
\hline Number of stabilization cycles & $10 \mathrm{cycles}$ \\
\hline Maximum stabilization time & $3.0 \mathrm{~s}$ \\
\hline Minimum stabilization cycle fraction & 0.00 \\
\hline
\end{tabular}




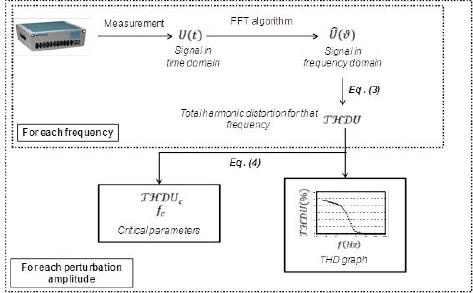




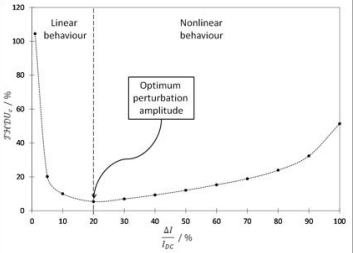




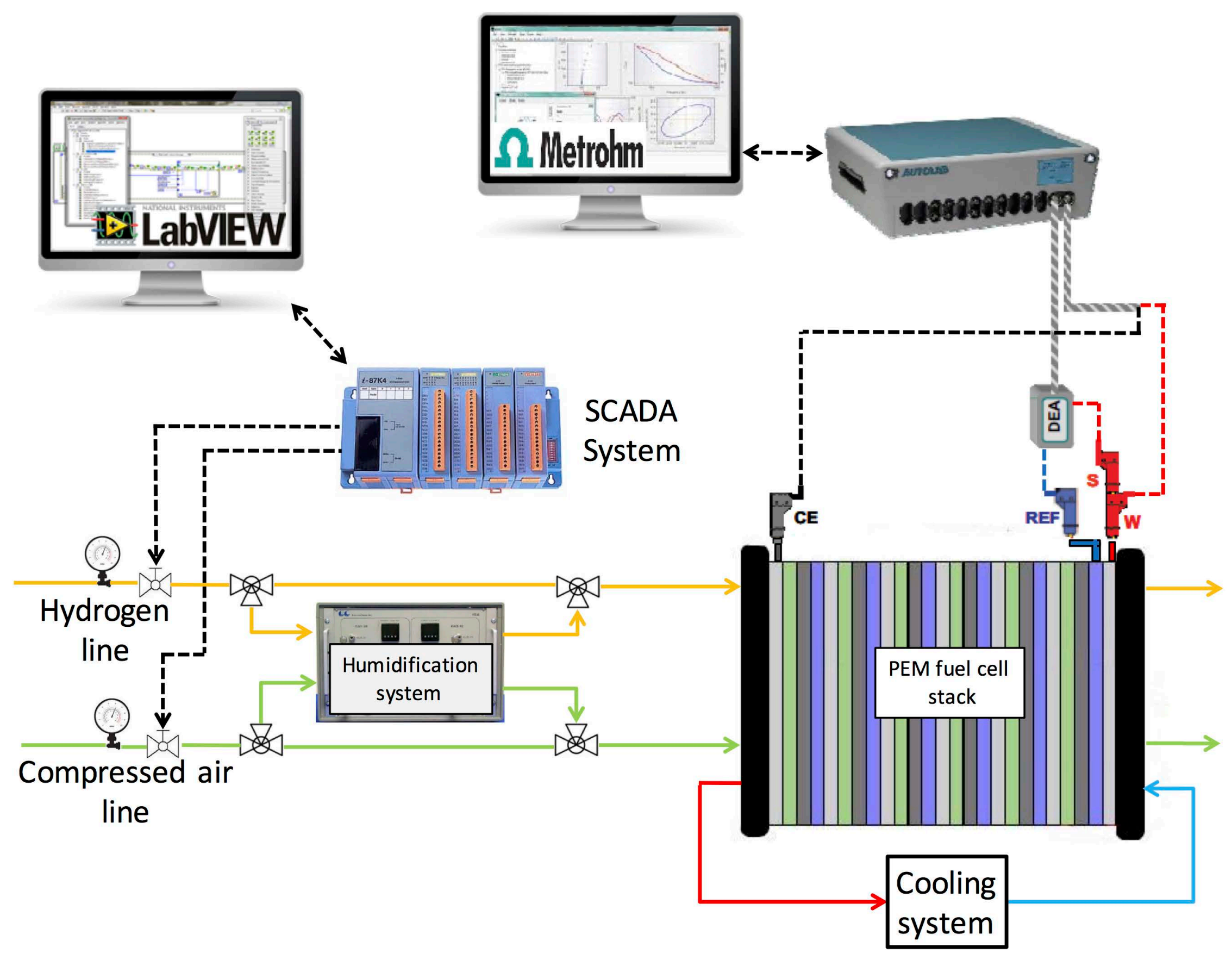




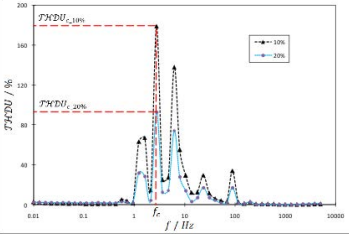




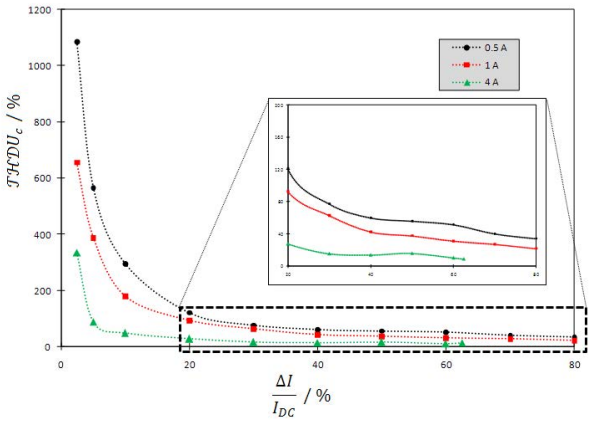




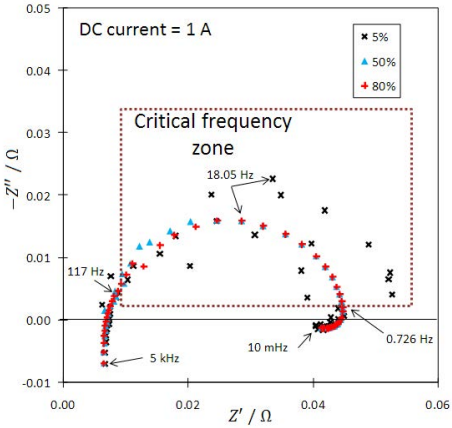




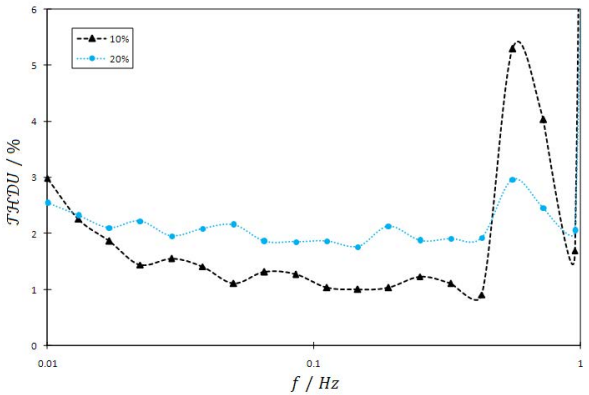




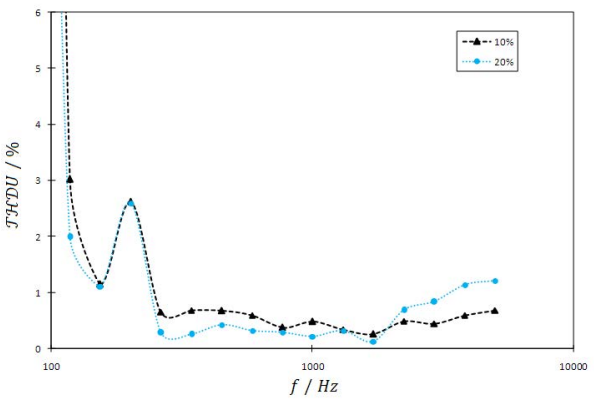




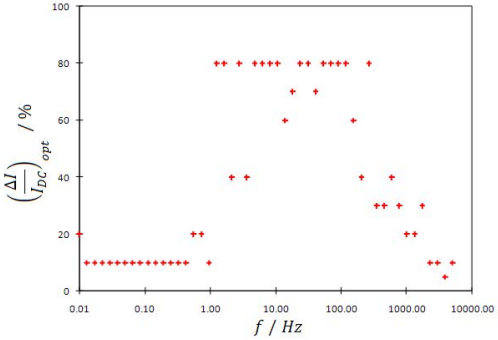




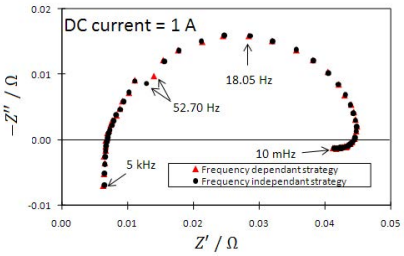

